\title{
Who Needs to be Informed? - Empirical Results From a Field Experiment on The Adoption of IOIS Among SMEs
}

\author{
Stanislav Kreuzer \\ Goethe-University Frankfurt \\ kreuzer@wiwi.uni-frankfurt
}

\section{Friedrich Born}

Goethe-University Frankfurt

fborn@wiwi.uni-frankfurt

\author{
Steffen Bernius \\ Goethe-University Frankfurt \\ bernius@wiwi.uni-frankfurt
}

\section{Abstract}

Inter-organizational information systems (IOIS) play a critical role in today's organizations and their relationships with business partners. While large organizations already began utilizing IOIS at the outset, small and medium-sized enterprises (SME) have subsequently been reluctant to adopt and use IOIS. As such systems are subject to high network effects, a firm thus has to reach out especially to its SME partners to achieve a critical mass of adopters among them. Prior research agrees that the provision of support in terms of circumstantial information and expertise can influence organizational adoption decisions. However, research in this direction has remained inconclusive. This study conducts a controlled field experiment at the organizational level to investigate the provision of support as a non-coercive persuasion strategy to foster the adoption of IOIS among 203 SME business partners of a large German organization. A cluster analysis is further conducted to identify distinct clusters of IOIS adopters showing significantly different adoption rates that result from informing them as a strategy. The results first offer evidence for the importance of informing SMEs as a viable strategy to foster IOIS adoption among them. Furthermore, the results provide empirical evidence for the presence of particular arrangements of characteristics describing the strategy and structure of analysed organizations that ultimately interact with the effect of the provision of support as a persuasion strategy.

Keywords: Inter-organizational information systems; organizational adoption; small and medium-sized enterprises; configuration analysis; persuasion strategy

\section{Introduction}

The adoption and use of inter-organizational information systems (IOIS) to conduct electronic business transactions across organizational borders is largely acknowledged to positively influence the financial and operational performance of today's organizations (Ke et al. 2013; Rai et al. 2006), as well as to generate considerable business value through IS integration and interoperability with business partners (Loukis and Charalabidis 2012). IOIS have existed for several decades (Robey et al. 2008), and have been successfully utilized in numerous contexts and industries such as electronic data exchange (EDI) (Massetti and Zmud 1996), electronic business-to-business (B2B) marketplaces (Howard et al. 2006), or Internet-based ordering platforms (Reimers et al. 2014). While large organizations began utilizing IOIS from their beginning, small and medium-sized enterprises (SME) have been largely reluctant about their use in the past (Iacovou et al. 1995), mainly due to the high setup and running costs of such systems, while presenting only limited benefits to SMEs (Kauffman and Mohtadi 2004). Even today, notwithstanding the fact that IOIS technology has vastly evolved from custom-built systems based on proprietary standards to Internet- and open standard-based systems (Zhu, Kraemer, Gurbaxani, et al. 2006), lowering the financial entry hurdle and thus making IOIS particularly valuable for SMEs, adoption and use of such systems among SMEs has remained low, with an average rate below 23\% (Raymond et al. 2013). For organizations considering 
adopting or which already have adopted a particular IOIS, this is especially troublesome as such systems are subject to high network effects, meaning that their value for each adopter largely depends on the total number of adopters using them ( $\mathrm{Zhu}$, Kraemer, Gurbaxani, et al. 2006). To realize the full potential of an IOIS implementation, a firm thus has to reach out especially to its SME business partners and achieve a critical mass of adopters among them (Au and Kauffman 2001).

Numerous studies have been conducted to understand the organizational IOIS adoption decisions of SMEs, and to present decision makers with persuasion strategies to foster adoption among SME business partners (Robey et al. 2008). Extant research introduced coercive as well as non-coercive strategies that organizations can follow to foster adoption among their business partners (Hart and Saunders 1997). For instance, Barua and Lee (1997) and Iacovou et al. (1995) identified the exertion of bargaining power (e.g. sanctioning undesired behaviour) as a strategy to coerce SME suppliers to adopt. In a similar vein, Iacovou et al. (1995) and Ramamurthy et al. (1999) introduced the provision of support (e.g. offering information and expertise, or financial and technical support) as a non-coercive strategy to increase awareness and technology readiness of SME suppliers and thus foster adoption among them. However, while the exerting of coercive power has been largely acknowledged as an effective strategy in this context (Teo et al. 2003), the strategy of providing support to SME business partners has received less attention from prior research (Son et al. 2008). As a review of that literature shows, investigations of the provision of support to SMEs have remained inconclusive. While several studies do report the provision of support to be a driver of organizational adoption in this context (Son et al. 2008), there are also studies arguing that the provision of support does not influence organizational decisions to adopt IOIS (Ulu and Smith 2009). Likewise, the review shows that experimental investigations of the provision of support have ranged from scarce to non-existent to date, leaving its causal relationship to organizational adoption of IOIS among SMEs experimentally unconfirmed. Finally, as the IOIS technology has been evolving dramatically over the last decade (Reimers et al. 2014), prior research can only profit from a critical re-examination in the light of the modern technology that is available for adoption by today's SMEs (Robey et al. 2008).

This study sets out to experimentally investigate the effects of providing support in terms of information and expertise to SME business partners as a non-coercive strategy to foster adoption of IOIS among them. Therefore, the study conducts a controlled field experiment among the SME business partners of a large German organization in its attempt to approach its partners and to foster the adoption of a particular IOIS through the provision of support in terms of circumstantial information and expertise. Thereby, only business partners categorized as SMEs according to the definition given by the European Union - namely, having less than 250 employees and an annual balance of less than 44 million Euro - are investigated and analysed (European Commission 2005). Acknowledging the need for a view beyond a single organization and towards groups of organizations operating in configurations of structure and strategy in research on the adoption of IOIS (Lyytinen and Damsgaard 2011), this study furthermore incorporates a configurational view of the organization in conducting its investigation that is also the predominant view in the domains of management and organizational research (Meyer et al. 1993; Miller 1986). A cluster analysis is applied to the experimental results as a suitable method for the analysis of configurations (Lyytinen and Damsgaard 2011), with the aim of identifying among the partners of the focal firm distinct groups or clusters of SMEs operating in different adopter configurations. Results of the experiment are then analysed in the light of the identified clusters. In so doing, this study addresses the following primary research questions:

RQ1: How does the provision of support in terms of circumstantial information and expertise by a focal firm to its SME business partners affect their organizational decisions towards the adoption of IOIS?

RQ2: How do differing adopter configurations, in which adopting SMEs are embedded and operate, affect an SME's susceptibility to the provision of support as a persuasion strategy? 
This study initially aims to observe an actual IOIS adoption in its natural environment. By drawing on prior research, a set of key characteristics for the adoption of SME business partners is further derived and utilized to identify distinct adopter configurations among the participating SMEs (Meyer et al. 1993). A cluster analysis is conducted as a suitable approach for the purpose of identifying distinct clusters pertaining to different adopter configurations among the set of derived key characteristics (Balijepally et al. 2011). An analysis of adoption among the identified adopter configurations then aims to uncover the influence that differing adopter configurations as such can have on the provision of support as a strategy to foster the adoption of IOIS among SMEs. Thereby, the study likewise aims to provide a possible explanation for varying results in prior research on the provision of support to SMEs. Finally, the results of this study inform decision makers on how to efficiently and effectively utilize the strategy of providing SME business partners with support by offering them information and expertise.

\subsection{The need for a focus on configurations of adopting organizations in research on IOIS adoption by SMEs}

Prior research on organizational IOIS adoption generally defines IOIS as information systems that are built around computer and communications technologies (e.g. personal computers, email), and are shared by at least two or more companies (J ohnston and Vitale 1988). Such information systems can be based on various technologies and standards (Zhu, Kraemer, Gurbaxani, et al. 2006). While in the past IOIS were largely based on proprietary systems (e.g. proprietary software), and proprietary standards such as EDI (Venkatraman and Zaheer 1990), modern IOIS however shift from using proprietary systems and standards to the use of open and Internet-based systems that are based on open standards such as XML (Reimers et al. 2014).

Prior investigations established an ample body of knowledge explaining organizational decisions towards adopting IOIS, and identified various adoption factors that describe aspects of the IOIS technology, the organization, as well as an organization's position in a particular business environment (Robey et al. 2008). These prior investigations often relied on Rogers' (1962) diffusion of innovations (DoI) theory to explain IOIS adoption decisions as optional innovation-decisions from a purely rationalistic perspective on adoption (Robey et al. 2008). In the context of IOIS adoption, DoI theory and the rationalistic perspective have therefore been criticized with regard to their ability to explain organizational adoption decisions in this context (Chau and Tam 1997). Specifically, Lyytinen and Damsgaard (2001) and Fichman (2004) criticize DoI's ignorance of the complex business environment in which organizations are embedded, as DoI's primary focus lies in examining singular technologies being adopted autonomously by individual adopters that are not embedded in complex networks. This limitation is likewise reported by Lee and Cheung (2004) in the context of IOIS adoption by SMEs, describing DoI as applying an individualist approach while ignoring the influence of organizational and environment factors.

Nevertheless, the domains of organizational and management research in particular value a focus on the organization and its environment, as the unit of analysis - the organization - is itself regarded as a set of tightly interconnected structures and practices (Fiss et al. 2013), continuously operating in a complex business environment and ever responding to its various characteristics (Bandiera et al. 2011). Management and organizational research thereby broadly acknowledge a configurational perspective in research of organizations that assumes that organizations operate in particular arrangements of organizational structure and strategy within their external business environment (Miller 1986). Organization theory further argues that organizations are best understood as organized clusters of interconnected factors rather than by analysing distinct components in isolation (Fiss 2007; Fiss et al. 2013), as one central observation in organizational analysis is that characteristics are often interdependent in essential ways (Meyer et al. 1993). Such organized clusters thereby can be described by configurations of conceptually distinct characteristics that commonly occur together and form an integrative and meaningful whole (Meyer et al. 1993). This observation is particularly valid 
in the case of IOIS adoption, as organizations that adopt IOIS are likewise seen to align along a limited set of coherent patterns of strategy and structure called adopter configurations according to characteristics describing their particular structural and strategic arrangements (Lyytinen and Damsgaard 2011).

Thus, investigations encompassing this interrelatedness can lead to more detailed insights into the mechanisms of organizational adoption of IOIS among SMEs and larger firms alike. For example, reported synergies between organizational and technological characteristics among business partners are able to provide an explanation for particular adoption decisions when benefits such as compatibility are perceived on both sides of a trading relationship (Venkatesh and Bala 2012). Similarly, reported moderation effects of organizational characteristics provide an explanation for variations in IOIS adoption decisions (Sila 2010). Therefore, it is argued that the particular adoption configuration an individual organization operates in is crucial in order to understand its adoption decision, for as a whole it may interact significantly with organizational adoption decisions and thereby provide valuable insights into why apparently structurally similar adopters make different decisions concerning IOIS adoption (Lyytinen and Damsgaard 2011).

\subsection{Informing SME partners as a key strategy to foster IOIS adoption among them}

An organization's decision to adopt a technological innovation is in most cases preceded by a high level of uncertainty (Fichman 2004). To counter this uncertainty, organizations tend to acquire circumstantial information about the innovation prior to the decision to adopt it (Rogers 1962). SMEs, however, differ from large firms in various ways (Thong 1999). SMEs reportedly suffer from limited information search and processing capabilities (Thong and Yap 1995), a lower level of awareness and a lack of IS knowledge and technical skills (Premkumar and Roberts 1999; Thong 1999), as well as limited financial resources and workforce (Thong 1999). Thus, a number of primary challenges have to be overcome by SMEs in order for them to be able to successfully adopt and use IT technologies such as IOIS.

The provision of support has been investigated in prior research as a strategy to overcome the aforementioned challenges of SMEs in the context of IOIS adoption, however these have yielded differing results in two primary research streams. One research stream relied on economic analysis and simulation to explain the effects of the provision of support to (nondominant) suppliers of a focal (dominant) firm. Riggins et al. $(1994,1995)$ investigated IOIS adoption among suppliers of a focal firm, suggesting that the provision of financial incentives to suppliers may foster adoption among them. Similarly, Nault et al. (1997) stress that besides financial incentives, the provision of information and expertise can contribute to successful IOIS adoption and implementation. On the contrary, Barua and Lee (1997) argue that the adoption decisions of suppliers to join an IOIS network are largely attributed to strategic necessity, based on the degree of their dependence on the focal firm. According to their analysis, neither financial incentives nor penalties influenced suppliers' adoption decisions. Mamer and McCardle (1987) and Ulu and Smith (2009) further argue that the provision of information does not necessarily lead to an increased intention to adopt an IOIS but can indeed lead to a more rapid decision on whether to adopt or reject it.

The other research stream relied on empirical investigations to study the influence of the provision of support on SMEs decisions to adopt IOIS, acknowledging that informing business partners about benefits and providing assistance in implementing the technology do present viable forms of persuasion strategies to foster adoption among them (Hart and Saunders 1997). Conducting an empirical study among seven companies, Iacovou et al. (1995) conclude that the provision of financial and technical assistance (e.g. offering information and technical expertise besides financial incentives) is able to foster adoption among SMEs. Similarly, Thong et al. (1996) present the results of a structural equation modelling (SEM) based analysis of survey results among 114 SMEs, and report that the provision of IS expertise from external consultants and technology vendors to SMEs is of critical importance as a means of fostering adoption of IOIS among SMEs. By analysing survey data from 194 SMEs utilizing SEM 
techniques, Raymond and Bergeron (1996) likewise report that cooperation received from the various business partners involved significantly influenced decisions of SMEs to adopt IOIS. Ramamurthy et al. (1999) analyse the responses from executives of 83 SMEs utilizing SEM techniques, and conclude that the provision of information and expertise is a key variable for influencing the IOIS adoption decisions of SME business partners, and thus suggest that firms provide greater support to partners who may not have the necessary expertise and knowledge. Khalifa and Davison (2006) analyse the data gathered from a survey among 114 SMEs utilizing SEM techniques, and find that the provision of support is among the significant predictors of organizational adoption in this context. Son et al. (2005) and Son et al. (2008) further provide empirical evidence for the influence of providing support to, and the degree of cooperation with, smaller suppliers on their adoption and use of IOIS. According to their investigations, this strategy is even more effective when long term relationships with a higher degree of cooperation are desired by business partners.

In summary, we make several key observations from reviewing prior investigations of the provision of support to SMEs. First, the review shows a fair amount of variability in results concerning the effect of the provision of support on organizational adoption decisions of SMEs. While economic investigations do not necessarily suggest the existence of a significant effect, empirical investigations nevertheless provide evidence for a significant influence of the provision of support on SMEs' adoption decisions in this context. Second, prior investigations have largely been ignorant of the possible interrelatedness of analysed characteristics that can be present in research on organizations (Fiss et al. 2013), primarily investigatingkey predictors as independent variables within statistical and economic models. Therefore, it is reasonable to suggest that the effect of the provision of support on SMEs' decisions to adopt IOIS could be dependent on the particular adopter configuration in which adoption decisions are made. Finally, experimental evidence for the causal relationship between the provision of support and SMEs' adoption of IOIS has remained scarce to non-existent to date. Nevertheless, particularly experimental investigations are essential to test and confirm causal relationships derived from theory and observation, as well as to assess the size of effects (Wade and Tingling 2005). Empirical investigations based on survey data are, however, limited in their ability to provide such results (Field and Hole 2003). In conclusion, we acknowledge the results of prior empirical analyses among SMEs and likewise expect the provision of support in terms of circumstantial information and expertise as a treatment within our experimental design to lead to a higher rate of adoption among participating SMEs. By increasing SMEs' awareness of IOIS (Thong 1999), counteracting their uncertainty connected with the adoption decision (Edelmann and Sintonen 2006; Thong et al. 1996), and further by positively affecting their technology readiness (Iacovou et al. 1995), we therefore propose that:

H1: $\quad$ Providing circumstantial information and expertise to SME business partners will lead to a higher rate of adoption among them

\subsection{Identifying key characteristics to describe the adopter configurations of SMEs}

A first step in the analysis of configurations and their influence on SMEs' adoption decisions is the identification of a set of key characteristics that are able to provide an integrative and meaningful picture of SME adopter configurations in the context of organizational IOIS adoption (Lyytinen and Damsgaard 2011). This set is likewise crucial for the cluster analysis, as the chosen variables define the structure of the dataset to be analysed (Balijepally et al. 2011). Prior research has investigated various characteristics describing organizational structure, as well as the business environment in the context of IOIS adoption (Robey et al. 2008), with several key characteristics being consistently reported to significantly influence organizational adoption decisions in this context. We focus on these characteristics in the following, assuming them to be the primary components likewise describing SMEs' configurations of organizational strategy and structure. 


\subsubsection{Characteristics describing organizational structure}

Organizational size is a key characteristic that has consistently been recognized as a driver of organizational innovation in general (Damanpour 1992), as well as in SMEs in particular (Thong and Yap 1995). Studies on IOIS adoption largely acknowledge its influence (Kauffman and Mohtadi 2004; Zhu, Kraemer, and Xu 2006). Similarly, organizational structure is likewise reported to affect organizational decisions in this context, as changes to organizational structure have to be made when adopting IOIS (Teo et al. 2006). Organizational structure thereby reflects the resource and routine rigidity of a firm and can ultimately prevent it from adopting an IOIS (Bala and Venkatesh 2007). Technology readiness, described as the extent to which technological capabilities and knowledge are present within the respective organization (Chwelos et al. 2001; Iacovou et al. 1995), is another key characteristic reported to significantly influence the adoption decisions of SMEs and larger firms alike (Thong 1999; Zhu, Kraemer, and $\mathrm{Xu}$ 2006).

\subsubsection{Characteristics describing organizational interaction with the external environment}

Characteristics describing environment related influence can generally be divided into five distinct types which are inherently distinct in the type of influence they have on organizational IOIS adoption (Kreuzer et al. 2014). Describing the influence of the business community on organizational decision making in this context, the type of industry of an organization has been reported to be of primary significance (Markus and Loebbecke 2013; Zhu, Kraemer, Gurbaxani, et al. 2006), as it is able to provide an adequate proxy for the particular strategic arrangement of an organization, mirroring the business environment in which it is embedded (Lyytinen and Damsgaard 2011). Theinfluence of business partners on organizational decision making is further described by the degree of interdependence between business partners as a primary characteristic (Hart and Saunders 1997). The differentiation of tiers has been investigated in this context to account for differences in interdependence (Iskandar et al. 2001). Furthermore, regulatory requirements can vary considerably between different regulatory environments (e.g. different countries), resulting in different decisions being made by otherwise similar organizations towards the adoption of IOIS (Teo et al. 2006). Therefore, the regulatory environment and particularly the regulatory scope of organizations are regarded as key characteristics in this context (Zhu, Kraemer, and Xu 2006). Relationship related influence further reflects the type of relationships that exist between a focal firm and its business partners through the degree of asset specificity as well as the duration of those relationships (Bala and Venkatesh 2007), with thelatter regarded as a key characteristic in this context (Venkatesh and Bala 2012). Finally, the particular institutional environment of a firm resulting from institutional forces that are present in its business environment can influence its decisions to adopt IOIS (DiMaggio and Powell 1983). Teo et al. (2003) describe such institutional forces to be primarily characterized by the types of a firm's competitors, customers, and suppliers.

\subsection{Identifying types of adopter configurations and their susceptibility to the strategy of informing}

Prior research on organizational IOIS adoption acknowledges the existence of frequently occurring structural arrangements in investigations of organizational IOIS adoption (Hart and Saunders 1997; Iacovou et al. 1995), as well as the ability of particular arrangements of structure and strategy to affect organizational decision making in this context (Lyytinen and Damsgaard 2011). Management research likewise acknowledges the existence of particular strategic arrangements - market-based and hierarchical setups - inside an organizational business landscape (Malone et al. 1987). Lyytinen and Damsgaard (2011) therefore identify four distinct types of organizational adopter configurations - dyadic, hub and spoke, industry, and community wide adopter configurations - as particular arrangements of structure and strategy that are able to affect organizational decision making. This configurational view is further supported by Albrecht et al. (2005), presenting a typology of distinct IOIS architectures, who argue that there is no single technology that provides a complete solution, 
as each is tailored to a particular strategy and purpose. In the following, we thus take a configurational view on organizations as forming organized clusters by operating in particular arrangements of organizational structure and strategy with their external environment (Meyer et al. 1993). We further argue that distinct types of such configurations are present in the business landscape (Lyytinen and Damsgaard 2011), and that these arrangements of inherently interrelated strategic and structural components as wholes are able to significantly influence the IOIS adoption decisions of SMEs (Meyer et al. 1993).

Dyadic configurations emerge when two autonomous organizations adopt an IOIS to create an electronic link for the exchange of business information (Chwelos et al. 2001). Configurations of this type generally mirror hierarchical strategic arrangements in supply chain contexts (Malone et al. 1987), with decision making likely to be largely defined by aspects of strategic necessity (Barua and Lee 1997), as well as interdependence (Pfeffer and Salancik 1978). Dyadic configurations are therefore likely to be employed primarily by SME firms manufacturing parts for higher tiers inside a supply chain context as a part of a hierarchical strategic arrangement (Iacovou et al. 1995). Furthermore, while SMEs in general are likely to have a lower level of awareness of the technology and a lack of IS knowledge and technical skills (Thong 1999), highly customized dyadic links and high asset specific investments can lead to modest routine (process) and resource (technical systems) rigidities in these SMEs that additionally impede information search and processing (Bala and Venkatesh 2007; Thongand Yap 1995). Similarly, such SMEs are likely to be oriented on long-term relationships with only a limited set of suppliers and customers (Klein and Rai 2009). For SMEs primarily operatingin configurations of a dyadic type, we therefore propose that the provision of information will have a positive effect, as these firms are likely to have a lower degree of awareness concerning new technologies and also limited information search and processing capabilities. These firms should therefore be eager to learn about them to increase competitiveness and optimize processes (Thong et al. 1996). The provision of information is thus able to increase SMEs' awareness of IOIS technology (Thong 1999), reduce the organizational uncertainty of SMEs connected with the adoption decision (Edelmann and Sintonen 2006; Thong et al. 1996), and increase the technology readiness of these firms (Iacovou et al. 1995), ultimately leading to a higher adoption rate among them. In conclusion, we hypothesize that:

H2: Providing circumstantial information and expertise to SME business partners primarily operating in adopter configurations of a dyadic type will lead to a higher rate of adoption among them

Hub and spoke configurations span particular industries (Lyytinen and Damsgaard 2011), and involve at least three adopters - two or more organizations that create an electronic link to a central hub by utilizing IOIS technologies (Koch and Schultze 2011). Such configurations are likely to be employed by final product assemblers and distributors that are either situated as a hub in a market-based, rather than a hierarchical, structure with their suppliers, or provide products to retail customers or other resellers on a similar basis (Howard et al. 2006; Reimers et al. 2014). Thus, SMEs operating in hub and spoke configurations can be characterized as the dominant buyers or suppliers in a market-type trading relationship that are less dependent on their business partners (Hart and Saunders 1997). Likewise, as they are situated in a marketbased structure with their partners, these firms are more interested in spreading their own IOIS (Howard et al. 2006), rather than adopting (eventually vastly different and incompatible) systems proposed by eventually less dominant partners (Markus and Loebbecke 2013). For SMEs primarily operating in hub and spoke configurations, we therefore propose that the provision of information will have only a minimal influence on their decision making as a result of them being only marginally interested in information about IOIS other than their own. Furthermore, rather than needing to be informed, these firms are more likely to provide information and expertise to their business partners themselves (Lyytinen and Damsgaard 2011). Concluding, we hypothesize that:

H3: Providing circumstantial information and expertise to SME business partners primarily operating in adopter configurations of a hub and spoke type will not lead to a higher rate of adoption among them 
Industry and community- wide configurations are described as emerging due to the concept of a common good, and to be based on mixes of adopter configurations of different types (Lyytinen and Damsgaard 2011). While industry configurations emerge inside an industry and are initiated by particular industry associations (Reimers et al. 2014), community-wide configurations emerge within a regional or national scope as a result of regional or national governmental IOIS initiatives (Penttinen et al. 2008). Such configurations largely depend on institutional involvement and therefore exhibit a more latent character (Lyytinen and Damsgaard 2011). Furthermore, they are likely to be populated by SMEs that operate within various and multiple business communities and industries, and are not bound to a supply chain context (Markus and Loebbecke 2013). SMEs situated in such configurations are therefore likely to adopt a "wait and see" approach to the adoption of IOIS (Edelmann and Sintonen 2006). This behaviour is based on the high organizational uncertainty resulting from SMEs' presence in multiple business communities, and of fears of failing to achieve a critical mass of business partners (Teo et al. 2006), or of adopting the wrong technology (Venkatesh and Bala 2012). The provision of circumstantial information and expertise is, however, not likely to reduce this uncertainty, as in this case the uncertainty largely stems from the multitude of divergent information sources available to SMEs in these configurations rather than from the absence of relevant information itself (Markus and Loebbecke 2013). This can even lead to an information overload when organizational information processing capabilities are low (Thong and Yap 1995), resulting in a perception of lower compatibility and higher complexity of IOIS (Kuan and Chau 2001). Concluding, we hypothesize that:

H4: Providing circumstantial information and expertise to SME business partners primarily operating in adopter configurations of an industry or community type will not lead to a higher rate of adoption among them

In summary, we expect that SMEs' decisions to adopt IOIS will be positively influenced overall by the provision of support to them in terms of circumstantial information and expertise. Nevertheless, the effect of the provision of support is expected to be significantly different among particular types of adopter configurations as a result of differences in strategic and structural arrangements.

\subsection{Empirical study}

The use of experiments is a widely acknowledged method to build, refine, accumulate, and apply knowledge in the social sciences (J ackson and Cox 2013). Experiments allow for the testing of causal relationships between independent and dependent variables, as well as assessing the size of effects (Wade and Tingling 2005). Laboratory experiments allow the researcher to create settings similar to their real-world counterparts through accurate simulation of real-world conditions in the laboratory (Grant and Wall 2009). However, the high degree of control does have a strong negative impact on the generalizability (external validity) of results (Kim et al. 2002; Winkler and Murphy 1973). On the other hand, field experiments are conducted within actual real-world settings under realistic conditions, and in so doing allow a balance between experimental control and generalizability (Harrison and List 2004). Furthermore, the field experiment has additional advantages when organizations are the unit of analysis. Decision-making in organizations is dependent on the particular external environment in which organizations are embedded and its characteristics which they have to respond and adapt to (Bandiera et al. 2011). Likewise, in most cases organizational decisionmaking is a cascaded process within a tightly interconnected corporate structure and is not subject to a single person (Meyer et al. 1993). Therefore, such organization-wide decisions are not testable in artificial laboratory conditions, even if the same participants would have been involved (Harrison and List 2004).

This study focuses the firm as its unit of analysis (e.g. the business partner firms of a focal organization). Thus, the field experiment was chosen as a suitable approach to analyse the posed research questions as it allows for the testing of the influence of a treatment in relation to a control group in a real-world scenario, which is not feasible by handing out questionnaires (Field and Hole 2003). Furthermore, the field experiment allows the observation and 
investigation of participants in their real-world environment, and likewise allows investigations of participants that are completely unaware that the experiment is taking place (Harrison and List 2004). The field experiment is thereby able to mitigate participant bias and achieve a much higher external validity of results (Field and Hole 2003; J ackson and Cox 2013). Nevertheless, there are also some downsides to conducting field experiments. Field experiments suffer from a limited control over key variables, difficulties in random assignment of participants to the different groups, as well as from difficulties in organizing and planning, as well as the high costs of such experimental designs for the field (Grant and Wall 2009; Kim et al. 2002). However, the downsides are addressed in the design of the conducted field experiment and are discussed in the following.

\subsubsection{Experimental setting}

As part of a long-term innovation strategy towards electronic document management and exchange, an automated electronic invoice processing system based on open-standards was adopted by the focal organization to optimize the invoice document flow from its business partners into the organization's internal ERP system. Invoice reception by the organization has been by paper only in the past for all of its business partners. Invoices were collected and periodically sent to a digitizing services provider where they were digitized to be integrated into the organization's ERP system. The new invoice processing system was able to handle electronic documents in an open-standard based portable document format (PDF) directly by extracting and restructuring the unstructured data of the invoices into the necessary form for integration into the target ERP system, allowing for a faster invoice processing. Thus, business partners, previously accustomed to a paper-based invoice process, were given the possibility of switching to an electronic means of direct invoice exchange by sending PDF invoices to the focal organization. The experimental design presented in the following was integrated into an address to the SME business partners of the focal organization. The aim of the focal organization thereby was to persuade business partners to switch to this new form of invoice exchange with the focal organization by applying a non-coercive persuasion strategy.

\subsubsection{Experimental design}

Adequate control of variables is a crucial concern particularly for field experimental designs (Grant and Wall 2009). Simple designs are therefore recommended when conducted within less controllable settings in the field (J ackson and Cox 2013). With this in mind, the design of the field experiment has been constructed to be as simple as possible. Thereby, we aimed to achieve a better control of the treatment, to minimize disruptions to the daily operations of the focal organization, and to minimize administrative complexity and costs. Furthermore, by designing a natural field experiment - a field experiment where the phenomenon of interest is investigated in its natural environment while participants are not aware of the experiment we aimed to maximize external validity (Harrison and List 2004). Therefore, the fieldexperimental design was placed within an ongoing IOIS implementation project of the focal organization, allowing the data collection to be conducted at the particular point in time when the focal firm was about to address its business partners. The questioned business partners thereby did not have to decide whether they could imagine adopting in the near future, but instead whether they actually would adopt or not. Furthermore, it was accurately ensured that the experiment would not be identified as such by the participating organizations. We made sure that only a small circle of people knew about the experiment while it was prepared and conducted. The group only involved several responsible managers of the focal organization, two consultants, and the authors. The field-experimental design consisted of one control group and one treatment group, with the treatment being the exogenous variable (Bandiera et al. 2011). The experimental design was integrated into an address of the focal organization to its business partners in the form of a cover letter. In this cover letter, the focal organization informed participants among its business partners of its novel ability to exchange electronic invoices. The cover letter further required the participating organizations to decide whether they would adopt it or not, and to reply to the cover letter stating their decision. Replies could either be sent by mail or fax, and a prepared reply sheet was provided in the cover letter. The reply sheet asked for a simple "yes" or "no" as a response regarding the adoption decision, as 
well as for a signature of a responsible contact person within the respective organization. The signature guaranteed that the replied decision was not a formless intention and the issue at hand had undergone a decision process within the respective organization, culminating in a signed answer in reply to the focal organization. As shown in figure 1, our control group received a cover letter from the focal organization, which did not have a high degree of information. In fact, the participation was stated as being completely voluntary and the degree of information can be understood as no different from a normal newsletter offering information about recent developments in a summarized form. No coercive pressure was applied within the cover letters of the respective groups. For our treatment group, we varied the exogenous variable to be circumstantial.

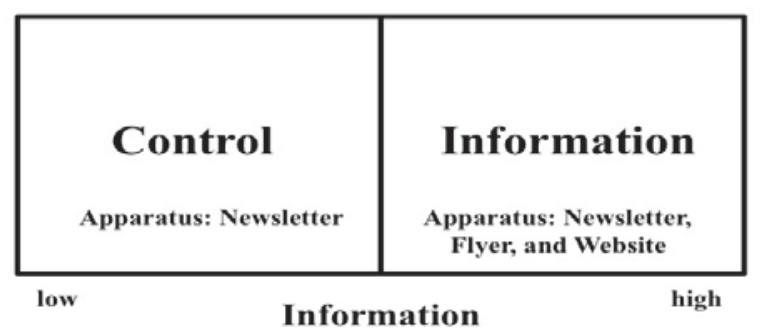

Figure 1: Treatment group, control group, and corresponding apparatus

The treatment group received a high degree of information by receiving a detailed cover letter, an additional brochure, and an additional link to a dedicated website giving specific and very detailed information about the technical solution and the potential benefits of the e-invoicing IOIS. While we could not control further research conducted by the subjects on the general topic of electronic invoicing, detailed information on this particular electronicinvoicing system was not publicly available. In order to prevent participants of the control group from finding the dedicated website, we prevented the usual search engine crawlers, like Google's, from indexing the homepage of the focal organization weeks in advance. After mailing the cover letter, participating organizations were given a timespan of four weeks to respond. Incoming electronic invoices without a preceding explicit reply were further accepted as a positive response. After the data collection period of four weeks, all participating organizations were debriefed with another cover letter including detailed information about the specific electronic invoicing system. The focal firm also distributed this information on their website. The participants did not face any repercussions by the focal organization after the end of the experiment.

\subsubsection{Participants}

In our experiment, 203 German SMEs were involved. All of them were business partners of the focal firm and could be categorized into what is often called B-class partners, defining them as important but not the most important ones. The focal organization is a firm with a longstanding tradition, established over 60 years ago, and employing over 1,500 people around the world. It is a private corporation with global operations, including more than a dozen manufacturing plants, and more than two dozen international offices, while doing business in 90 countries. We chose only business partners with at least a shallow relationship to the focal firm, which was assessed through the amount of invoices exchanged per year. The minimum of received invoices per year has been set to three, while the upper limit was set to a 100 invoices per year. On the one hand, the selected group of SMEs accounted for a third of total annually received invoices, while it still offered a wide range of relationship strength through the frequency of annual invoice volume as a proxy. Most of the subjects were suppliers of raw materials, manufactured goods, or miscellaneous services. All subjects in the group were further categorized as SMEs according to the definition given by the European Commission, having less than 250 employees and an annual balance of less than 44 million Euros (European Commission 2005). This approach is acknowledged by prior research, with studies providing definitions of SMEs primarily relying on employee counts and annual balance information as 
limiting criteria, and applying country-specific limits (Thong and Yap 1995; Chwelos et al. 2001). In the European case, criteria provided by the European Commission (2005) thus are regarded as a suitable approach. However, we had to relax the definition to assert at least one requirement to be fulfilled (using or conjunction), as annual balance information was not always available. Larger business partners have been addressed separately and also personally during the migration to electronic invoicing.

To ensure an adequate random assignment of participating organizations among the experimental groups in the field experiment, retained organizations were randomly distributed among the treatment and control groups. Nevertheless, we introduced several constraining rules to avoid an uneven distribution of either large or small firms, and an uneven distribution of related industries. Likewise, to ensure that the dependence relationship with the focal firm would not bias experimental results, the random assignment was controlled with respect to the amount of invoices exchanged with the focal firm per year as an adequate proxy for the level of dependence in the relationship (Chwelos et al. 2001). After the random assignment, we checked the resulting group structures and compared them to the overall sample structure. This resulted in two almost equal groups as can be seen in Table 1.

\begin{tabular}{|l|c|c|c|}
\hline & Information & Control & Total \\
\hline Sample size & 105 & 99 & 203 \\
\hline Average invoice frequency per year & 15 & 15 & 15 \\
\hline Median of invoice frequency per year & 8 & 8 & 8 \\
\hline Range of invoice frequency per year & $3-96$ & $3-100$ & $3-100$ \\
\hline
\end{tabular}

Table 1: Sample profile

We made sure, that both groups were heterogeneous within while being homogenously structured compared to each other. The probability of receiving a certain treatment or no treatment at all was equal for each subject, which resulted in a randomized sample. With almost equal structural groups, it could then be assumed that the exogenous treatments were responsible for the difference in outcomes (Bandiera et al. 2011).

\subsubsection{Operationalization of the key characteristics for the analysis of adopter configurations}

To be able to identify and analyse different adopter configurations, the identified key characteristics were operationalized as categorical variables, and objective observational data was collected for all participating organizations accordingly. All variables describing the characteristics were transformed onto a categorical scale, if needed, to mitigate otherwise present scale differences across clustering variables (Balijepally et al. 2011).

- The organizational size of SMEs was measured through a categorical variable describing the number of employees (Thong 1999). SMEs were described as having "1 to 9", "10 to 49", "50 to 199" and "200 and more" employees.

- $\quad$ Organizational structure was measured by a dichotomous variable describing an organization either as having a "centralized" organization structure or a "distributed" organization structure, based on the availability and focus on subsidiaries.

- Technology readiness was measured by a categorical variable describing the availability and the capabilities of the corporate website (Hong and Zhu 2006). This approach provided a suitable proxy for the assessment of technological capabilities and thus technological readiness of firms in the Internet age (Zhu and Kraemer 2002). Technology readiness was described as "low" when no or only a basic website was available, "moderate" when a timely and up-to-date website was available, and "high" when a timely website was available which also offered dynamic interaction capabilities 
(e.g. web-shop or a dynamic communication platform such as chat-based customer support).

- $\quad$ Business community influence was measured by a categorical variable describing the type of industry in which an organization operates, differentiating between "parts manufacturing", "final assembly / wholesale", and "service provision" (Hong and Zhu 2006).

- $\quad$ Business partner-related influence was measured by a categorical variable describing the degree of interdependence between the organization and its business partners. Interdependence was "high" for organizations situated within a supply chain context (e.g. organizations manufacturing parts for partners of higher tiers), "moderate" at the end of a supply chain context (e.g. final assemblers or resellers), and "low" when an organization was not situated in a supply chain context (e.g. service provision, banks) (Iskandar et al. 2001).

- The influence of the regulatory environment was measured through the scope of operation of an organization, being either "regional", "national", or "international" in nature (Zhu, Kraemer, and Xu 2006).

- $\quad$ Relationship-related influence was measured by a categorical variable describing the degree of importance of the relationship for a particular organization as having a focus on "long term relationships", a focus on "occasional customers", or "no focus" (Venkatesh and Bala 2012).

- Institutional forces were measured through two categorical variables describing the primary industry type of suppliers and customers of an organization (Teo et al. 2003). Suppliers were described as being of type "resource supply", "parts supply", or "no primary type". Customers were described as being of type "manufacturing", "reseller", or "no primary type".

Data for the operationalized variables was collected from openly available data sources such as commercial and trade registers, the participating companies' webpages, industry associations, and recent news. The data was evaluated by a four-headed research team and used for the subsequent cluster analysis.

\subsubsection{Cluster analysis of adopter configurations}

Cluster analysis is regarded as a powerful approach for extracting natural configurations among data that otherwise cannot be easily seen by the naked eye (Hair et al. 2013). In the context of IOIS adoption, Lyytinen and Damsgaard (2011) therefore likewise argue that, when taking a configurational perspective, cluster analysis is an approach well-suited to uncovering natural configurations among adopting organizations according to a set of key characteristics that describe the particular configurations of structure and strategy in which organizations are embedded. Thus, by drawing from current theoretical conceptions on the appropriate use of cluster analysis as a method in IS research (Balijepally et al. 2011), we utilize cluster analysis to analyse SMEs' adopter configurations according to previously derived key characteristics.

The process of cluster analysis can generally be conducted according to several critical steps (Hair et al. 2013). The first step of the process is the selection of objectives and the selection of clustering variables. In our case, the objective of the analysis was to identify IOIS adopter configurations as clusters in our data, and attribute each of them to one of the generic IOIS adopter configuration types. To this end, we derived a set of key characteristics from prior research on IOIS adoption. The next step of the process was the standardization of variables. As the identified key characteristics consisted of metric, nominal and ordinal attributes, we operationalized all key characteristics on the nominal and ordinal scale. Furthermore, to mitigate any scale differences between different ordinal scales, we defined the ordinal characteristics to be treated in the same way as nominal scale characteristics (disregarding order information), thus further standardizing all key characteristics to be treated equally. The hamming distance (MacWilliams and Sloane 1977), a measure for similarity or dissimilarity 
between two data objects with categorical attributes, was chosen as a suitable distance measure in the next step. To adequately handle the purely categorical space and produce proper results utilizing the hamming distance, we further selected a clustering algorithm developed for the purpose of clustering categorical spaces (Zhang et al. 2006). The selected algorithm does not require any specific statistical models or assumptions about particular underlying statistical distributions of cluster attributes, as it utilizes a Pearson chi-squared-type statistic to identify clusters among the datasets based on hamming distance vectors. The partition of the produced dataset is further insensitive to the input order of the datasets, making it also invariable in relation to particular starting solutions (Hair et al. 2013). The algorithm further identifies one cluster per iteration, excluding it from the dataset for the next iteration, and thus is able to determine the number of clusters automatically.

\section{Results}

The field experiment was started in the last week of April 2013. We collected the data over a course of four weeks after we sent out the different treatments by mail. Table 2 shows the responses of our participants. We gathered the positive and negative responses of each group, which have been the dichotomous variables. Our results show that between the treatment group and the control group, there is no significant effect of the treatment on the rate of adoption among organizations $(\mathrm{p}=0.862, \varphi=0.02)$. Therefore, no support for hypothesis $\mathrm{H} 1$ could be found.

\begin{tabular}{|r|c|c|}
\hline & Treatment & Control \\
\hline Yes & 37 & 32 \\
\hline No & 31 & 30 \\
\hline No Answer & 37 & 36 \\
\hline p value & \multicolumn{2}{|c|}{0.862} \\
\hline Effect size & \multicolumn{2}{|c|}{0.020} \\
\hline
\end{tabular}

Table 2: Overview of the treatment effects in the full dataset

\subsection{Results of the cluster analysis}

Participating SMEs were clustered among the set of identified key characteristics to address the remaining hypotheses. The cluster analysis resulted in four distinct clusters presented in Table 3.

The cluster centre of each cluster thereby represents the most characteristic configuration for values of the respective characteristics in this cluster. In so doing, the cluster centres describe the characteristic adopter configuration of SMEs in their respective cluster among the set of key characteristics. Based on the characteristic configuration of key characteristics, cluster 1 thereby represents an adoption configuration of a hub and spoke type, comprising primarily small (characteristic count of $9<\mathrm{x}<50$ employees for firms in cluster 1 according to the SME definition of the European Commission (2005)) SMEs that focus long term relationships and operate as hubs for international suppliers or customers. Accordingly, cluster 2 can be attributed to the dyadic adopter configuration type, comprising primarily medium-sized (characteristic count of $49<\mathrm{x}<250$ employees for firms in cluster 2 according to the SME definition of the European Commission (2005)) manufacturing SMEs that primarily operate within highly interdependent dyadic supply chains that cross national borders. Cluster 3 can be attributed to a community-based adopter configuration type, comprising primarily microsized (based on the characteristic count of $0<\mathrm{x}<10$ employees for firms in cluster 3 according to the SME definition of the European Commission (2005)) SME service providers operating on national level and providing services within various configurations and therefore not having a primary supplier or customer type. One dataset was further grouped into a fourth cluster. 
However, as one dataset is not enough to identify a particular adopter configuration type, the dataset is marked as an outlier.

\begin{tabular}{|r|c|c|c|c|}
\hline Dataset Count & Cluster 1 & Cluster 2 & Cluster 3 & Outliers \\
\hline Industry Type & $\begin{array}{c}\text { final assembly / } \\
\text { wholesale }\end{array}$ & $\begin{array}{c}\text { parts } \\
\text { manufacturing }\end{array}$ & $\begin{array}{c}\text { service } \\
\text { provision }\end{array}$ & - \\
\hline Organizational Size & 10 to 49 & 50 to 199 & 1 to 9 & - \\
\hline Regulatory Scope & international & international & national & - \\
\hline $\begin{array}{r}\text { IT Readiness } \\
\text { Organizational } \\
\text { Structure }\end{array}$ & moderate & moderate & moderate & - \\
\hline $\begin{array}{r}\text { Relationship } \\
\text { Importance }\end{array}$ & $\begin{array}{c}\text { long term } \\
\text { relationships }\end{array}$ & $\begin{array}{c}\text { long term } \\
\text { relationships }\end{array}$ & $\begin{array}{c}\text { long term } \\
\text { relationships }\end{array}$ & - \\
\hline Interdependence & moderate & high & low & - \\
\hline Primary Supplier Type & parts supply & resource supply & no primary type & - \\
\hline Primary Customer Type & reseller & manufacturing & no primary type & - \\
\hline
\end{tabular}

Table 3: Overview of the clusters in the dataset, and their respective cluster centres

To test hypotheses H2-H4, we conducted Fisher's exact test for count data to analyse the significance of the association between the administration of our treatment and the rate of adoption among SMEs in the treatment and the control groups of the respective clusters (Fisher 1925). Furthermore, we computed a $\times 2$ statistic to obtain a measure of the effect size $\varphi$ of our treatment (Cramer's V) (Cramér 1946). The effect size can thereby be described as small, medium, and large for $\varphi$ values greater than $0.10,0.30$, and 0.50 respectively (Field and Hole 2003). The resulting contingency tables for the control and treatment groups of the three clusters, each counting the replies of SMEs in respect to their decision to adopt or reject the IOIS, are shown in the following Table 4.

\begin{tabular}{|c|c|c|c|c|c|c|}
\hline & \multicolumn{2}{|c|}{ Cluster 1} & \multicolumn{2}{|c|}{ Cluster 2} & \multicolumn{2}{|c|}{ Cluster 3} \\
\hline & Treatment & Control & Treatment & Control & Treatment & Control \\
\hline Yes & 10 & 12 & 23 & 9 & 4 & 11 \\
\hline No & 10 & 11 & 8 & 12 & 13 & 7 \\
\hline No Response & 12 & 11 & 10 & 13 & 14 & 12 \\
\hline p value (two-tailed) & \multicolumn{2}{|c|}{1.0} & \multicolumn{2}{|c|}{$\mathbf{0 . 0 4 0 8} *$} & \multicolumn{2}{|c|}{$\mathbf{0 . 0 4 0 9} *$} \\
\hline$\chi^{2}$ statistic & \multicolumn{2}{|c|}{0.020} & \multicolumn{2}{|c|}{5.194} & \multicolumn{2}{|c|}{5.042} \\
\hline effect size $\varphi$ & \multicolumn{2}{|c|}{0.022} & \multicolumn{2}{|c|}{0.316} & \multicolumn{2}{|c|}{0.380} \\
\hline
\end{tabular}

Table 4: Overview of the treatment effects in the three clusters

Our results show that in the case of cluster 1, representing a hub and spoke adopter configuration, there is no significant effect of the treatment on the rate of adoption among SMEs in this cluster $\left(p=1.0, \chi^{2}=0.014, \varphi=0.017\right)$. Therefore, no support for hypothesis $\mathrm{H}_{3}$ could be found. For cluster 2, representing a dyadic adopter configuration, our results show a significant positive effect of medium size for the treatment on the rate of adoption among SMEs in this cluster $\left(\mathrm{p}=0.0409, \chi_{2}=5.194, \varphi=0.316\right)$, thereby supporting hypothesis $\mathrm{H} 2$. In the case of cluster 3 , our results further show a significant negative effect of medium size for the treatment on the rate of adoption among SMEs in this cluster $(p=0.0409, \chi 2=5.042, \varphi=0.380)$, thereby supporting hypothesis H4. 


\section{Discussion}

According to the results, when treating all participants equally, the provision of support in terms of circumstantial information and expertise as a persuasion strategy resulted in no significant change in SMEs' adoption rates (no support for H1). Furthermore, adoption seemed to be modest at best, with nearly half of the responding SMEs refusing to adopt. However, significant effects of moderate size could be uncovered for the strategy of providing support among particular adopter configurations of participating SMEs.

In the case of the dyadic-type configuration, the provision of support in our field experiment led to a significant, medium-sized positive effect regarding the adoption rate among SME business partners (support for H2). This result suggests that the provision of circumstantial information and expertise indeed increases the low awareness of SMEs in this group that results from only limited information search and processing capabilities by relieving them from the actual search task (Thong and Yap 1995). Furthermore, present organizational uncertainty is reduced through the provision of circumstantial information (Thong et al. 1996). Finally, technology readiness of SMEs is increased by enhancing their understanding of and expertise in the technology (Iacovou et al. 1995). The result is then a higher adoption rate of IOIS among the respective SMEs. This explanation is further supported by the only moderate degree of IT readiness and the high degree of interdependence with suppliers and customers present among SMEs in this group, suggesting that the provision of information compensates the only limited information search and processing capabilities. SMEs in this group, however, are eager to learn about new technologies to increase competitiveness and to optimize processes (dyadic electronic exchanges) with business partners.

In the case of the identified configuration of a hub and spoke type, no significant effect could be observed regarding the adoption rate in this group (no support for H3). This result nevertheless suggests that organizations in this configuration do not seem to be significantly affected by the provision of support in terms of circumstantial information and expertise. A possible explanation for this result could be that SMEs operating as hubs for suppliers and customers already possess an adequate level of awareness and technology readiness, and are not affected by the provision of already known (redundant) information and expertise (Markus and Loebbecke 2013). Furthermore, SMEs operating as hubs may be more interested in convincing their business partners to adopt their own solution, rather than otherwise (Howard et al. 2006). The only moderate degree of interdependence in this group likewise suggests that SMEs in this group are less dependent on their business partners and may be more interested in promoting their own IOIS, as well as informing business partners themselves (Lyytinen and Damsgaard 2011).

In the case of the identified community-type configuration, the provision of information led to a significant negative effect of medium size regarding the adoption rate among firms in this group (support for H4). This result not only shows that SMEs in this group display a "wait and see" approach towards the adoption of IOIS that is not significantly mitigated through the provision of information and expertise (Edelmann and Sintonen 2006), but more importantly also suggests that the provision of information does indeed lead to an intensified perception of the IOIS as being more complex and less compatible by SMEs in this group, ultimately leading to the opposite effect of a lower adoption rate. This perception is thereby unlikely to be a result of an absence of awareness or information, but rather a result of the amount and diversity of information available to SMEs in this group (Markus and Loebbecke 2013). Accordingly, in this group the low organizational information processing capabilities of SMEs lead to an overload of information (Thong and Yap 1995), resulting in the IOIS being perceived as more complex and less compatible (Kuan and Chau 2001). The low degree of interdependence and the absence of a primary supplier and customer types likewise suggest that SMEs in this group are operating in highly heterogeneous business environments. Thus, SMEs may not be willing to bind themselves to a particular IOIS due to a fear of failing to achieve a critical mass of business partners (Teo et al. 2006), or adopting the wrong technology (Venkatesh and Bala 2012). 
In summary, the results of the conducted field experiment did not provide empirical support for an overall positive effect of the provision of support in terms of circumstantial information and expertise as a persuasion strategy to foster SMEs' adoption of IOIS. A cluster analysis, however, was able to uncover several adopter configurations of differing types that represent differing arrangements of strategy and structure in which adopting SMEs operate. These differing types of adopter configurations exhibit a significant variation in adoption rates, and provide a possible explanation for variations in prior investigations of the influence of the provision of support on SMEs' adoption decisions in this context.

\section{Conclusion and Further Research}

Our objective in this study was to examine, how the provision of circumstantial information and expertise can be used as an effective persuasion strategy to foster the adoption of IOIS among SME business partners of a focal firm. By conducting a controlled field experiment, we were able to observe an actual IOIS adoption taking place in its natural environment. A cluster analysis of a set of identified key characteristics of participating SMEs and their environment in our field experiment further was able to explain how the provision of support as a persuasion strategy is able to affect decisions to adopt IOIS of SMEs operating in particular adoption configurations, thus answering our research question RQ1. By analysing the results of our experiment in the light of the identified adopter configurations, we were further able to uncover and explain differing susceptibilities of structurally similar SMEs situated in different adopter configurations to the strategy of the provision of support, thus answering our research question RQ2. Our results show that the strategy of informing SME business partners about an IOIS to foster the adoption among them is not necessarily beneficial, but can be a vital strategy to foster adoption among SMEs operating in particular adoption configurations. However, it can also hinder the adoption in SMEs operating in particular others.

\section{Theoretical and Practical Contribution}

This study uncovers contrary decisions of structurally similar SMEs by analysing their decision to adopt in the light of their operation in different configurations of strategy and structure. These differences would have been overlooked by a traditional analysis without a focus on adoption configurations. The analysis that was carried out to test H1 likewise supports this, as it did not find any significant effect of the treatment in the experimental setup. Nevertheless, significant effects are indeed present (H2, H4) when focusing on differing adoption configurations within the analysis. Thus, this study contributes to the existing body of knowledge by first providing experimental evidence for the causal relationship between the provision of support to, and the organizational IOIS adoption by, SMEs. Second, the study provides empirical evidence for the importance of the analysis of adopter configurations to enable better explanations of organizational IOIS adoption by SMEs. In particular, this study provides an important insight for investigations on this factor of influence, suggesting that future research pay attention to the presence of arrangements of characteristics describing the strategy and structure of analysed organizations, as these are able to significantly interact with the effect of the provision of support as a persuasion strategy. Such multilateral interactions thus provide a possible explanation for varying results in prior studies, and furthermore might provide even better explanations of organizational IOIS adoption when focused in future investigations. Finally, this study provides future studies with a set of key characteristics derived from prior research that are able to provide a meaningful picture of configurations of organizational structure and strategy in the context of IOIS adoption by SMEs.

Besides the theoretical contribution, the results of this study are also of interest to organizations that plan to or already have adopted an IOIS, and now are attempting to foster its adoption among business partners through the strategy of providing support in terms of information and expertise. This study uncovers several distinct clusters of organizations that decide differently when information is provided as a persuasion strategy. While business partners operating within dyadic adopter configurations are quite susceptible to the strategy of informing, business partners operating in community-based adopter configurations will 
exhibit a quite contrary behaviour, when confronted with circumstantial information. Business partners operating within adopter configurations of a hub and spoke type will furthermore not be significantly affected by the strategy of informing. The results of this study suggest that, particularly in supply chain contexts, the innovativeness of SMEs is significantly suppressed through the high levels of customization present in dyadic links. Nevertheless, the provision of information and expertise is particularly effective for SMEs operating in supply chain contexts, as these SMEs are eager to innovate, while not being overwhelmed by the information provided. The results also provide decision makers with an approach to identify business partners that are in need of information and expertise to be able to adopt a particular IOIS. Decision makers can utilize this approach to provide support only where it is needed, and likewise are able to increase the effectiveness of this strategy by not overloading the wrong partners with redundant information.

\subsection{Limitations and Further Research}

This study is subject to several limitations that have to be acknowledged. First, based on the relatively short time span of the field experiment of four weeks, we had to use a proxy for adoption in our experimental design, since not every business partner had sent an invoice during this period (this can also be due to the fact that roughly half of participating SMEs might send only eight or fewer invoices per year to the focal organization, meaning that the mean period between invoices would be six weeks). Adoption was therefore measured through formal replies that had to be signed. We thereby assumed that business partners of the focal firm would take the signed agreement seriously. Furthermore, key characteristics identified in the course of this study were operationalized as categorical, and were all analysed on the nominal scale. Thereby, information was lost in several characteristics to a certain degree. The rationale for this approach was to achieve a standardization of all variables prior to conducting a cluster analysis (Hair et al. 2013). However, future research should investigate alternate methods of identifying adopter configurations to avoid losing information for the sake of standardization. Similarly, we acknowledge that the identified key characteristics in our study do not present an exhaustive set of attributes for the identification of adopter configurations. Therefore, subsequent research should focus on uncovering additional key characteristics in this context. Furthermore, by investigating only non-coercive persuasion, and analysing only SMEs that have not been forced to adopt IOIS, this study focused on only one of the two primary persuasion strategies identified in prior research (Son et al. 2008). Future investigations in this direction should analyse both strategies - providing support and exerting coercive force - in conjunction so as to provide richer insights and implications for research and practice. Questions arising from the results of this study in the context of organizational IOIS adoption could for example investigate which particular adopter configurations are susceptible to which particular strategy, or analyse if the application of both strategies will lead to better results.

\section{References}

Albrecht, C. C., Dean, D. L. \&Hansen, J . V. (2005) “Marketplace and technology standards for B2B e-commerce: progress, challenges, and the state of the art", Information \& Management, 42(6):865-875.

Au, Y. A. \& Kauffman, R. J . (2001) "Should We Wait? Network Externalities, Compatibility, and Electronic Billing Adoption", Journal of Management Information Systems, 18(2):47-63.

Bala, H. \& Venkatesh, V. (2007) “Assimilation of Interorganizational Business Process Standards", Information Systems Research, 18(3):340-362.

Balijepally, V., Mangalaraj, G. \& Iyengar, K. (2011) "Are We Wielding this Hammer Correctly? A Reflective Review of the Application of Cluster Analysis in Information Systems Research", J ournal of the Association for Information Systems, 12(5):375-413. 
Bandiera, O., Barankay, I. \& Rasul, I. (2011) "Field Experiments with Firms", The J ournal of Economic Perspectives, 25(3):63-82.

Barua, A. \& Lee, B. (1997) "An Economic Analysis of the Introduction of an Electronic Data Interchange System", Information Systems Research, 8(4):398-422.

Chau, P. Y. K. \& Tam, K. Y. (1997) "Factors Affecting the Adoption of Open Systems: An Exploratory Study", Management Information Systems Quarterly, 21(1):1-24.

Chwelos, P., Benbasat, I. \& Dexter, A. S. (2001) "Research Report: Empirical Test of an EDI Adoption Model”, Information Systems Research, 12(3):304-321.

Cramér, H. (1946) Mathematical methods of statistics, Princeton University Press, Princeton.

Damanpour, F. (1992) “Organizational Sizeand Innovation”, Organization Studies, 13(3):375402.

DiMaggio, P. J . \& Powell, W. W. (1983) "The Iron Cage Revisited: Institutional Isomorphism and Collective Rationality in Organizational Fields", American Sociological Review, 48(2):147-160.

Edelmann, J . \& Sintonen, S. (2006) "Adoption of electronic invoicing in Finnish SMEs: two complementary perspectives", International Journal of Enterprise Network Management, 1(1): 79-98.

European Commission (2005) The new SME definition - User guide and model declaration, European Commission, Brussels.

Fichman, R. G. (2004) "Going Beyond the Dominant Paradigm for Information Technology Innovation Research: Emerging Concepts and Methods", J ournal of the Association for Information Systems, 5(8):314-355.

Field, A. P. \& Hole, G. (2003) How to design and report experiments, Sage Publications, London; Thousand Oaks.

Fisher, R. A. (1925) Statistical methods for research workers, Oliver \& Boyd, Edinburgh, London.

Fiss, P. C. (2007) "A Set-Theoretic Approach to Organizational Configurations", The Academy of Management Review, 32(4):1180-1198.

Fiss, P. C., Marx, A. \& Cambré, B. (2013) "Configurational Theory and Methods in Organizational Research: Introduction", in Configurational Theory and Methods in Organizational Research, Research in the Sociology of Organizations, P. C. Fiss, B. Cambré \&A. Marx (eds.), 38:1-22, Emerald Group Publishing Limited, Bingley.

Grant, A. M. \& Wall, T. D. (2009) "The Neglected Science and Art of Quasi-Experimentation: Why-to, When-to, and How-to Advice for Organizational Researchers", Organizational Research Methods, 12(4):653-686.

Hair, J . F., Black, W. C., Babin, B. J . \& Anderson, R. E. (2013) Multivariate Data Analysis, Pearson Education Limited, Harlow.

Harrison, G. W. \& List, J . A. (2004) "Field Experiments”, J ournal of Economic Literature, 42(4):1009-1055.

Hart, P. \& Saunders, C. (1997) "Power and Trust: Critical Factors in the Adoption and Use of Electronic Data Interchange”, Organization Science, 8(1):23-42.

Hong, W. \& Zhu, K. (2006) "Migrating to internet-based e-commerce: Factors affecting ecommerce adoption and migration at the firm level", Information \& Management, 43(2):204-221. 
Howard, M., Vidgen, R. \& Powell, P. (2006) "Automotive e-hubs: Exploring motivations and barriers to collaboration and interaction", J ournal of Strategic Information Systems, 15(1):51-75.

Iacovou, C. L., Benbasat, I. \& Dexter, A. S. (1995) "Electronic Data Interchange and Small Organizations: Adoption and Impact of Technology", Management Information Systems Quarterly, 19(4):465-485.

Iskandar, B. Y., Kurokawa, S. \& LeBlanc, L. J. (2001) "Adoption of Electronic Data Interchange: The Role of Buyer-Supplier Relationships", IEEE Transactions on Engineering Management 48(4):505-517.

J ackson, M. \&Cox, D. R. (2013) 'The Principles of Experimental Design and Their Application in Sociology", Annual Review of Sociology, 39(1):27-49.

J ohnston, H. \& Vitale, M. (1988) “Creating Competitive Advantage with Interorganizational Information Systems”, Management Information Systems Quarterly, 12(2):153-165.

Kauffman, R. J . \& Mohtadi, H. (2004) "Proprietary and Open Systems Adoption in EProcurement: A Risk-Augmented Transaction Cost Perspective", Journal of Management Information Systems, 21(1):137-166.

Ke, W., Liu, H., Wei, K. \& Hua, Z. (2013) "Supply Chain Exploitation, Exploration, and Firm Performance: Effects of Top Management and Information Technology Capabilities", in Proceedings of the 34th International Conference on Information Systems (ICIS), December 15-18, Milano, Italy.

Khalifa, M. \& Davison, R. N. (2006) "SME adoption of IT: the case of electronic trading systems", IEEE Transactions on Engineering Management, 53(2):275-284.

Kim, B., Barua, A. \&Whinston, A. B. (2002) "Virtual field experiments for a digital economy: a new research methodology for exploring an information economy", Decision Support Systems, 32(3):215-231.

Klein, R. \& Rai, A. (2009) "Interfirm Strategic Information Flows in Logistics Supply Chain Relationships", Management Information Systems Quarterly, 33(4):735-762.

Koch, H. \& Schultze, U. (2011) "Stuck in the Conflicted Middle: A Role-Theoretic Perspective on B2B E-Marketplaces", Management Information Systems Quarterly, 35(1):123-146.

Kreuzer, S., Krönung, J . \& Bernius, S. (2014) “Dismantling The Environmental Context - The Role of Environmental Characteristics in The Organizational Adoption of Open Standard-Based Inter-Organizational Information Systems", in Proceedings of the 22nd European Conference on Information Systems (ECIS), J une 5-13, Tel Aviv, Israel.

Kuan, K. K. Y. \& Chau, P. Y. K. (2001) “A perception-based model for EDI adoption in small businesses using a technology- organization- environment framework", Information \& Management, 38(8):507-521.

Lee, M. K. O. \& Cheung, C. M. K. (2004) "Internet Retailing Adoption by Small-to-Medium Sized Enterprises (SMEs): A Multiple-Case Study", Information Systems Frontiers, 6(4):385-397.

Loukis, E. \& Charalabidis, Y. (2012) “Business Value of Information Systems Interoperability - A Balanced Scorecard Approach", in Proceedings of the 20th European Conference on Information Systems (ECIS), J une 10-13, Barcelona, Spain.

Lyytinen, K. \& Damsgaard, J. (2001) “What's Wrong with the Diffusion of Innovation Theory?", in Diffusing Software Product and Process Innovations, IFIP - The International Federation for Information Processing, M. A. Ardis and B. L. Marcolin (eds.), 173-190, Springer, Berlin; Heidelberg. 
Lyytinen, K. \& Damsgaard, J . (2011) "Inter-organizational information systems adoption - a configuration analysis approach", European Journal of Information Systems, 20(5):496-509.

MacWilliams, F. J . \& Sloane, N. J . A. (1977) The theory of error-correcting codes, Elsevier, Amsterdam.

Malone, T. W., Yates, J. \& Benjamin, R. I. (1987) "Electronic markets and electronic hierarchies", Communications of the ACM, 30(6):484-497.

Mamer, J . W. \& McCardle, K. F. (1987) 'Uncertainty, Competition, and the Adoption of New Technology”, Management Science, 33(2):161-177.

Markus, M. L. \& Loebbecke, C. (2013) "Commoditized Digital Processes and Business Community Platforms: New Opportunities and Challenges for Digital Business Strategies", Management Information Systems Quarterly, 37(2):649-654.

Massetti, B. \& Zmud, R. W. (1996) "Measuring the Extent of EDI Usage in Complex Organizations: Strategies and Illustrative Examples", Management Information Systems Quarterly, 20(3):331-345.

Meyer, A. D., Tsui, A. S. \&Hinings, C. R. (1993) “Configurational Approaches to Organizational Analysis", The Academy of Management J ournal, 36(6):1175-1195.

Miller, D. 1986. "Configurations of strategy and structure: Towards a synthesis", Strategic Management J ournal, 7(3):233-249.

Nault, B. R., Wolfe, R. A. \& Dexter, A. S. (1997) "Support strategies to foster adoption of interorganizational innovations", IEEE Transactions on Engineering Management, 44(4):378-389.

Penttinen, E., Dorota, K., Harald, B., Hyytiäinen, M., Kuivalahti, T., Poteri, J . \& Turunen, T. (2008) Electronic Invoicing Initiatives in Finland and in the European Union - Taking the Steps Towards the Real-Time Economy, Helsinki School of Economics, Helsinki.

Pfeffer, J. \& Salancik, G. R. (1978) The external control of organizations: a resource dependence perspective, Harper \& Row, New York.

Premkumar, G. \&Roberts, M. (1999) "Adoption of newinformation technologies in rural small businesses", Omega, 27(4):467-484.

Rai, A., Patnayakuni, R. \& Seth, N. (2006) "Firm Performance Impacts of Digitally Enabled Supply Chain Integration Capabilities", Management Information Systems Quarterly, 30(2):225-246.

Ramamurthy, K., Premkumar, G. \& Crum, M. R. (1999) “Organizational and Interorganizational Determinants of EDI Diffusion and Organizational Performance: A Causal Model", Journal of Organizational Computing and Electronic Commerce, 9(4):253-285.

Raymond, L. \& Bergeron, F. (1996) "EDI success in small and medium-sized enterprises: A field study", J ournal of Organizational Computing and Electronic Commerce, 6(2):161172.

Raymond, L., Bergeron, F. \& Croteau, A. M. (2013) "Innovation Capability and Performance Of Manufacturing SMEs: The Paradoxical Effect of IT Integration", Journal of Organizational Computing and Electronic Commerce, 23(3):249-272.

Reimers, K., J ohnston, R. B. \& Klein, S. (2014) "An empirical evaluation of existing IS change theories for the case of IOIS evolution", European Journal of Information Systems, 23(4):373-399. 
Riggins, F. J ., Kriebel, C. H. \& Mukhopadhyay, T. (1994) "The Growth of Interorganizational Systems in the Presence of Network Externalities", Management Science, 40(8):984998.

Riggins, F. J., Mukhopadhyay, T. \& Kriebel, C. H. (1995) “Optimal policies for subsidizing supplier interorganizational system adoption", J ournal of Organizational Computing, 5(3):295-325.

Robey, D., Im, G. \& Wareham, J . (2008) 'Theoretical Foundations of Empirical Research on Interorganizational Systems: Assessing Past Contributions and Guiding Future Directions", J ournal of the Association for Information Systems, 9(9):497-518.

Rogers, E. M. (1962) Diffusion of Innovations, Free Press, New York.

Sila, I. (2010) "Do organisational and environmental factors moderate the effects of Internetbased interorganisational systems on firm performance?", European Journal of Information Systems, 19(5):581-600.

Son, J .-Y., Narasimhan, S. \& Riggins, F. J . (2005) "Effects of Relational Factors and Channel Climate on EDI Usage in the Customer-Supplier Relationship", J ournal of Management Information Systems, 22(1):321-353.

Son, J .-Y., Narasimhan, S., Riggins, F. J . \& Kim, N. (2008) “Understanding the Development of IOS-Based Trading Partner Relationships: A Structural Model with Empirical Validation", J ournal of Organizational Computing and Electronic Commerce, 18(1):3460.

Teo, H. H., Wei, K. K. \&Benbasat, I. (2003) “Predicting Intention to Adopt Interorganizational Linkages: An Institutional Perspective", Management Information Systems Quarterly, 27(1):19-49.

Teo, T. S. H., Ranganathan, C. \& Dhaliwal, J . (2006) "Key Dimensions of Inhibitors for the Deployment of Web-Based Business-to-Business Electronic Commerce", IEEE Transactions on Engineering Management, 53(3):395-411.

Thong, J. Y. L. (1999) "An Integrated Model of Information Systems Adoption in Small Businesses", J ournal of Management Information Systems, 15(4):187-214.

Thong, J. Y. L., Yap, C.-S. \& Raman, K. S. (1996) “Top Management Support, External Expertise and Information Systems Implementation in Small Businesses", Information Systems Research, 7(2):248-267.

Thong, J. Y. \& Yap, C.-S. (1995) "CEO characteristics, organizational characteristics and information technology adoption in small businesses", Omega, 23(4):429-442.

Ulu, C. \& Smith, J. E. (2009) "Uncertainty, Information Acquisition, and Technology Adoption", Operations Research, 57(3):740-752.

Venkatesh, V. \& Bala, H. (2012) "Adoption and Impacts of Interorganizational Business Process Standards: Role of Partnering Synergy", Information Systems Research, 23(4):1131-1157.

Venkatraman, N. \& Zaheer, A. (1990) "Electronic Integration and Strategic Advantage: A Quasi-Experimental Study in the Insurance Industry", Information Systems Research, 1(4):377-393.

Wade, M. R. \&Tingling, P. (2005) "A NewTwist on an Old Method: A Guide to the Applicability and Use of Web Experiments in Information Systems Research", Database, 36(3):6988.

Winkler, R. L. \& Murphy, A. H. (1973) "Experiments in the laboratory and the real world", Organizational Behavior and Human Performance, 10(2):252-270. 
Zhang, P., Wang, X. \& Song, P. X.-K. (2006) “Clustering Categorical Data Based on Distance Vectors", J ournal of the American Statistical Association, 101(473):355-367.

Zhu, K. \& Kraemer, K. L. (2002) "e-Commerce Metrics for Net-Enhanced Organizations: Assessing the Value of e-Commerce to Firm Performance in the Manufacturing Sector", Information Systems Research, 13(3):275-295.

Zhu, K., Kraemer, K. L., Gurbaxani, V. \& Xu, S. X. (2006) "Migration to Open-Standard Interorganizational Systems: Network Effects, Switching Costs, and Path Dependency", Management Information Systems Quarterly, 30(SI):515-539.

Zhu, K., Kraemer, K. L. \& Xu, S. (2006) "The Process of Innovation Assimilation by Firms in Different Countries: A Technology Diffusion Perspective on E-Business", Management Science, 52(10):1557-1576.

Copyright: (c) 2015 Kreuzer, Born, Bernius. This is an open-access article distributed under the terms of the Creative Commons Attribution-NonCommercial 3.0 Australia License, which permits non-commercial use, distribution, and reproduction in any medium, provided the original author and AJ IS are credited.

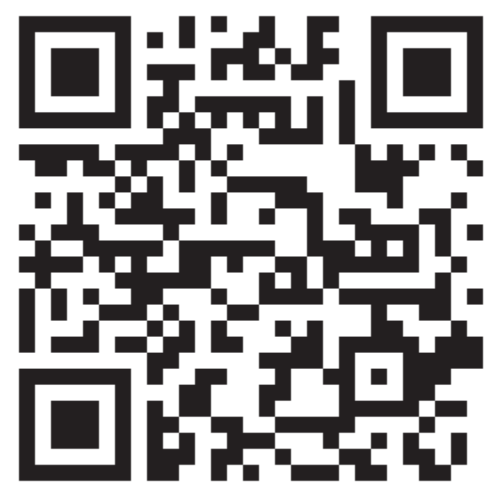

